\title{
Changing priorities in investor decision-making: the sustainability agenda
}

Cath Jackson, University of Sheffield Allison Orr, University of Glasgow 


\section{Introduction}

$\square$ Momentum driving the Sustainability Agenda is increasing

$\square$ Notable policy and legislative changes:

- EU directives - 2009 and others

- UN Global Compact (world's largest corporate sustainability initiative)

- RICS (2015) Advancing Responsible Business Practices in Land, Construction and Real Estate Use and Investment

$\square$ Policy goals:

- To embed sustainable and inclusive practices in the global economy

- To create a sustainable built environment at its centre

- Embrace human rights, labour, environment and anti-corruption principles 


\section{Investors}

$\square$ Identified as key stakeholder group by RICS (2015)

$\square$ Responsibilities span across all three phases of the life-cycle of real estate assets

- Development; Use; Recovery

$\square$ Important/strategic role in pursuing more sustainable strategies and practices for land and buildings

$\square$ Yet, little known about their attitudes/behaviour or if/how sustainable strategies and targets shape their decisionmaking 


\section{What we do know ...}

$\square$ Corporate responsibility has been developing and is being embraced in investment strategies

$\rightarrow$ Socially Responsible Investment (SRI) strategies:

"characterise the behaviour of investors who not only focus on the mere economic aspects of an investment but also follow ethical principles and take into account environmental and social aspects" (Lorenz and Lützkendorf, 2008, p. 483)

"efforts that go beyond compliance with minimum legal requirements to better manage the environmental, social, and governance issues associated with property investing" (Pivo, 2008, p. 235)

(3) RICS

Research

Trust 


\section{... and in practice ...}

\section{User markets:}

- Sustainable buildings no longer seen as "inconveniences"

- Can $\uparrow$ productivity and $\downarrow$ costs

$\square$ US: Energy Star/LEED Star rating attracts rent premia (Eichholtz et al., 2010; Fuerst and McAllister,2011; Wiley, 2010)

- Australia: Green Star ratings yield higher rent (Newell et al., 2011)

Netherlands: lower EPC = lower rents (Kok and Jennen, 2012)

$\square$ UK: less clear

- Fuerst and McAllister (2011) no evidence (but appraisal data)

- Chegut et al. (2012) 23-30\% for BREEAM rated buildings in London

- Fuerst and van de Wetering (2015) BREEAM 23\% to 26\% higher (across UK but year dependent)

Overall: magnitude of premia varies (3\% to 30\%), office sector common focus 


\section{... and in practice ...}

\section{Investment markets:}

- US: CV premia (6\%-29\%) for green-rated offices (Eichholtz et al., 2010; Miller et al., 2008; Pivo and Fisher, 2010; Fuerst and McAllister, 2011)

- Australia: Newell et al. (2011) find $12 \%$ premia

$\square$ UK: Chegut et al. (2011) find 27-43\% premia for BREEAM rated buildings

$\square$ Fuerst (2015) - tentative evidence that REITs return-on-asset and returnon-equity increase as sustainability rating increases 


\section{... and in practice ...}

\section{Investment markets:}

- US: CV premia (6\%-29\%) for green-rated offices (Eichholtz et al., 2010; Miller et al., 2008; Pivo and Fisher, 2010; Fuerst and McAllister, 2011)

- Australia: Newell et al. (2011) find 12\% premia

$\square$ UK: Chegut et al. (2011) find 27-43\% premia for BREEAM rated buildings

$\square$ Fuerst (2015) - tentative evidence that REITs return-on-asset and returnon-equity increase as sustainability rating increases

- Some evidence of improved occupancy rates (Pivo and Fisher, 2010; Fuerst and McAllister, 2011)

$\square$ Some evidence of reduced operational costs (Pivo and Fisher, 2010)

$\square$ Little (formal) progress on appraisal methods 


\section{... and in practice ...}

\section{Investment markets:}

- US: CV premia (6\%-29\%) for green-rated offices (Eichholtz et al., 2010; Miller et al., 2008; Pivo and Fisher, 2010; Fuerst and McAllister, 2011)

- Australia: Newell et al. (2011) find $12 \%$ premia

$\square$ UK: Chegut et al. (2011) find 27-43\% premia for BREEAM rated buildings

$\square$ Fuerst (2015) - tentative evidence that REITs return-on-asset and returnon-equity increase as sustainability rating increases

$\square$ Some evidence of improved occupancy rates (Pivo and Fisher, 2010; Fuerst and McAllister, 2011)

$\square$ Some evidence of reduced operational costs (Pivo and Fisher, 2010)

$\square$ Little (formal) progress on appraisal methods

$\square$ Overall: if rental premia priced by market, what are investment drivers?

(5) RICS

Research

Trust 


\section{Research Question}

$\square$ Sustainability agenda:

- Two elements: (i) management \& policy

(ii) implementation \& measurement

- Latter "is a vastly more significant and more powerful driver of financial performance" yet Europe scores comparatively low (Fuerst, 2015, p. 13)

$\square$ Alignment of financial returns / environmental concerns?

$\square$ No substantive evidence on investors' responses in practice 


\section{Research Question}

$\square$ Sustainability agenda:

- Two elements: (i) management \& policy

(ii) implementation \& measurement

- Latter "is a vastly more significant and more powerful driver of financial performance" yet Europe scores comparatively low (Fuerst, 2015, p. 13)

$\square$ Alignment of financial returns / environmental concerns?

$\square$ No substantive evidence on investors' responses in practice

Do investors' preferences and behaviour align with policy and stated sustainability objectives?

(3) Rics

Research

Trust 


\section{Research Design}

\section{Mixed methods approach:}

$\square$ Stage 1 - Conjoint Analysis/Questionnaire

- Simulate complex decision-making process

- Replicate heterogeneous nature of real estate assets

- Additional contextual questions

- Observe behaviour

$\square$ Stage 2 - Focus Groups

- 2 to 3 meetings with key stakeholders, semi-structured

- In-depth $\rightarrow$ views, experiences, policies, drivers for/ barriers to implementation 


\section{Conjoint Analysis}

$\square$ Choice-Based Conjoint (CBC) Analysis

$\square$ Primary data collection

$\square$ Temporal - 2006/07 and 2016

$\square 2016$ online

$\square$ Method

- Choice between two real estate assets (or choose neither)

- Eight property attributes

- Each asset comprises randomised levels of each attribute

- Tasks repeated

- Opportunity to segment and control for sector and economic outlook 


\begin{tabular}{|c|c|c|}
\hline Attributes & & $\begin{array}{c}\text { Levels } \\
\end{array}$ \\
\hline \multirow{4}{*}{ Credit worthiness } & 1) & Minimum risk of tenant default \\
\hline & 2) & Lower than average risk of tenant default \\
\hline & 3) & Higher than average risk of tenant default \\
\hline & 4) & High risk of tenant default \\
\hline \multirow{3}{*}{ Single or multi-let } & 1) & Single let property \\
\hline & 2) & 2 to 5 tenants \\
\hline & 3) & More than 5 tenants \\
\hline \multirow{5}{*}{ Rent review clause } & 1) & Rent set annually, linked to index or turnover \\
\hline & 2) & Rent review every 2 to 3 years, upwards only clause \\
\hline & 3) & Rent review every 4 or more years, upwards only clause \\
\hline & 4) & Rent review every 2 to 3 years, no upwards only clause \\
\hline & 5) & Rent review every 4 or more years, no upwards only clause \\
\hline \multirow{3}{*}{ Period to expiry/break } & 1) & Less than 5 years \\
\hline & 2) & $5-10$ years \\
\hline & 3) & Over 10 years \\
\hline \multirow{3}{*}{ User/Assignment clause } & 1) & Restrictive user/assignment clause \\
\hline & 2) & Standard user/assignment clause \\
\hline & 3) & Relaxed or no user/assignment clause \\
\hline \multirow{6}{*}{ Location } & 1) & Town or city centre, prime pitch \\
\hline & 2) & Town or city centre, secondary pitch \\
\hline & 3) & Suburban location, close to existing public transportation \\
\hline & 4) & Suburban location, no existing public transportation \\
\hline & 5) & Out of the town/city, close to existing public transportation \\
\hline & 6) & Out of the town/city, no existing public transportation \\
\hline \multirow{6}{*}{ BREEAM rating } & 1) & Pass \\
\hline & 2) & Good \\
\hline & 3) & Very Good \\
\hline & 4) & Excellent \\
\hline & 5) & Outstanding \\
\hline & 6) & Not known \\
\hline \multirow{3}{*}{$\begin{array}{l}\text { Economic and functional } \\
\text { obsolescence }\end{array}$} & 1) & High spec and flexible internal configuration \\
\hline & 2) & Average spec and internal configuration \\
\hline & 3) & Low spec and inflexible internal configuration \\
\hline
\end{tabular}




\section{Analysis}

$\square$ Counting analysis

- Relative impact of each attribute level

- Identify main effects and interaction effects

- Simplistic (but useful) method

$\square$ Multinomial logit estimation

- Relative importance of attributes

- Difference each attribute could make to the total utility of asset

- Estimate the utility (part-worths) of each level of each attribute

$\square$ Hierarchical Bayes (HB) estimation

- More robust - (part-worths) calculated for each respondent (Count \& ML averages) 


\section{Samples}

\begin{tabular}{|r|c|c|}
\hline Return Objective & 2006/07 Funds & 2016 Funds \\
Income return & $9.8 \%$ & \\
Capital growth return & $9.8 \%$ & $21.7 \%$ \\
Income \& capital return & $76.5 \%$ & $58.5 \%$ \\
Other return objective & $3.9 \%$ & $7.3 \%$ \\
\hline Vehicle Style & & \\
Core/Core+ & $49.0 \%$ & $80.5 \%$ \\
Value added & $15.7 \%$ & $4.9 \%$ \\
Opportunistic & $15.7 \%$ & $4.9 \%$ \\
Other style & $17.6 \%$ & $9.5 \%$ \\
Track & $3.9 \%$ & \\
Outperform & $58.8 \%$ & $58.5 \%$ \\
Split & $11.8 \%$ & $0.0 \%$ \\
Benchmark Objective & $0.0 \%$ & $31.7 \%$ \\
Obsolute return & $3.9 \%$ & $7.3 \%$ \\
\hline Other objective & & \\
Open-ended & $76.5 \%$ & $73.2 \%$ \\
Closed & $21.6 \%$ & $26.8 \%$ \\
\hline Type of Fund & & \\
\hline
\end{tabular}




\section{Samples}

\begin{tabular}{|r|c|c|}
\hline Return Objective & 2006/07 Funds & 2016 Funds \\
Income return & $9.8 \%$ & $31.7 \%$ \\
Capital growth return & $9.8 \%$ & $2.4 \%$ \\
Income \& capital return & $76.5 \%$ & $58.5 \%$ \\
Other return objective & $3.9 \%$ & $7.3 \%$ \\
\hline Vehicle Style & & \\
Core/Core+ & $49.0 \%$ & $80.5 \%$ \\
Value added & $15.7 \%$ & $4.9 \%$ \\
Opportunistic & $15.7 \%$ & $4.9 \%$ \\
Other style & $17.6 \%$ & $9.5 \%$ \\
\hline Track & $3.9 \%$ & \\
Benchmark Objective & & $2.4 \%$ \\
Outperform & $58.8 \%$ & $58.5 \%$ \\
Absolute return & $11.8 \%$ & $0.0 \%$ \\
Other objective & $0.0 \%$ & $31.7 \%$ \\
Open-ended & $3.9 \%$ & $7.3 \%$ \\
Closed & $21.6 \%$ & $26.8 \%$ \\
\hline Type of Fund & & \\
\hline
\end{tabular}

\begin{tabular}{|r|c|c|}
\hline & $\mathbf{2 0 0 6 / 0 7}$ & $\mathbf{2 0 1 6}$ \\
\hline Male & $90.2 \%$ & $92.7 \%$ \\
Female & $9.8 \%$ & $7.1 \%$ \\
\hline Experience in current role & & \\
0 to 5 years & $21.6 \%$ & $39.0 \%$ \\
6 to 10 years & $17.6 \%$ & $34.1 \%$ \\
$>10$ years & $60.8 \%$ & $26.8 \%$ \\
\hline Highest qualification & & \\
BSc/BA or equivalent & $52.9 \%$ & $65.9 \%$ \\
MSc/ MA or equivalent & $31.4 \%$ & $29.3 \%$ \\
PhD/Mphil & $3.9 \%$ & $2.4 \%$ \\
Other & $11.8 \%$ & $2.4 \%$ \\
\hline Total No Respondents & 51 & 41 \\
\hline
\end{tabular}


Location

In town or city centre

Town or city centre, prime pitch

Town or city centre, secondary pitch

Suburban, close to existing public transportation

Suburban, no existing public transportation

Out of the town/city, close to existing public transportation

Out of the town/city, no existing public transportation Within Att. Chi-Square

Single or multi-let

Single let property

2 to 5 tenants

More than 5 tenants

Within Att. Chi-Square

\section{Economic and functional obsolescence}

High specification and flexible internal configuration

Average specification and internal configuration

Low specification and inflexible internal configuration

Within Att. Chi-Square

\section{Rent review clause}

Rent set annually, linked to index or turnover

Rent review every 2 to 3 years, upwards only clause

Rent review every 4 or more years, upwards only clause

Rent review every 2 to 3 years, no upwards only clause

Rent review every 4 or more years, no upwards only clause

Within Att. Chi-Square
0.52

\section{BREEAM rating}

\begin{tabular}{cc} 
& 0.43 \\
& 0.18 \\
0.38 & 0.25 \\
0.19 & 0.08 \\
0.38 & 0.19 \\
0.18 & 0.08 \\
90.66 & 117.34 \\
\hline
\end{tabular}

\begin{tabular}{|c|c|}
\hline 0.22 & 0.19 \\
\hline 0.37 & 0.20 \\
\hline 0.4 & 0.21 \\
\hline 33.86 & 0.55 \\
\hline
\end{tabular}
$0.55^{*}$
$0.47 \quad 0.29$

$0.34 \quad 0.21$

$0.18 \quad 0.11$

80.09
41.27 \begin{tabular}{|l|l|}
\hline 0.34 & 0.21 \\
\hline
\end{tabular}

\begin{tabular}{l|l}
0.37 & 0.21
\end{tabular}

\begin{tabular}{l|l}
0.36 & 0.19
\end{tabular}

$0.27 \quad 0.21$

\begin{tabular}{l|l}
0.29 & 0.19
\end{tabular} $8.91^{*} \quad 1.07^{*}$
Period to expiry/break $3.83^{*} \quad 88.01$

\section{User/assignment clause}

\begin{tabular}{|c|c|c|}
\hline Over 10 years & 0.40 & 0.23 \\
\hline Within Att. Chi-Square & 16.13 & $3.98^{*}$ \\
\hline
\end{tabular}

\begin{tabular}{|r|c|c|}
\hline Restrictive user/assignment clause & 0.30 & 0.20 \\
\hline Standard user/assignment clause & 0.34 & 0.20 \\
\hline Relaxed or no user/assignment clause & 0.35 & 0.21 \\
\hline Within Att. Chi-Square & $3.31^{*}$ & $0.09 *$ \\
\hline
\end{tabular}

\section{Credit worthiness of the tenant}

D\&B 5AA rating for tenant(s) $\quad 0.38$

Minimum risk of tenant default

D\&B 3AA or 4AA rating for tenant(s) $\quad 0.37$

Lower than average risk of tenant default

0.25

D\&B $1 A A$ or 2AA rating for tenant(s) $\quad 0.33$

Higher than average risk of tenant default

$D \& B A A$ or $B B$ or $C C$ rating for tenant(s)

0.32

$D \& B$ DD or lower rating for tenant(s)

0.25

High risk of tenant default

Within Att. Chi-Square

12.17 


\begin{tabular}{|c|c|c|c|c|c|}
\hline \multicolumn{6}{|c|}{ Attribute Level Utilities and Preferences (based on ML Estimation) Across the Total Sample } \\
\hline & $2006 / 07$ & 2016 & & 2006/07 & 2016 \\
\hline Location & & & BREEAM rating & & \\
\hline In town or city centre & 0.99 & & Pass & $0.00 *$ & -0.66 \\
\hline Town or city centre, prime pitch & & 1.31 & Good & $-0.16 *$ & $0.07^{*}$ \\
\hline Town or city centre, secondary pitch & & $-0.04 *$ & Very Good & 0.28 & 0.43 \\
\hline Suburban, close to existing public transportation & 0.33 & 0.41 & Excellent & $-0.01 *$ & $0.22 *$ \\
\hline Suburban, no existing public transportation & -0.77 & -0.78 & Outstanding & & 0.32 \\
\hline Out of the town/city, close to existing public transportation & 0.36 & $0.02 *$ & Not known & $-0.11 *$ & -0.39 \\
\hline Out of the town/city, no existing public transportation & -0.91 & -0.93 & & & \\
\hline Single or multi-let & & & Period to expiry/break & & \\
\hline Single let property & -0.56 & $-0.04 *$ & Less than 5 years & -0.29 & -0.20 \\
\hline 2 to 5 tenants & 0.2 & $-0.01 *$ & 5-10 years & $-0.01 *$ & $0.02 *$ \\
\hline More than 5 tenants & 0.36 & $0.05^{*}$ & Over 10 years & 0.3 & 0.18 \\
\hline Economic and functional obsolescence & & & User/assignment clause & & \\
\hline High specification and flexible internal configuration & 0.69 & 0.51 & Restrictive user/assignment clause & -0.22 & 0.02 \\
\hline Average specification and internal configuration & 0.16 & $0.04^{*}$ & Standard user/assignment clause & $0.07^{*}$ & 0.00 \\
\hline Low specification and inflexible internal configuration & -0.84 & -0.55 & Relaxed or no user/assignment clause & 0.15 & $-0.02 *$ \\
\hline Rent review clause & & & Credit worthiness of the tenant & & \\
\hline Rent set annually, linked to index or turnover & $0.05^{*}$ & $0.00 *$ & D\&B 5AA rating for tenant(s) & 0.33 & \\
\hline Rent review every 2 to 3 years, upwards only clause & 0.35 & $0.02 *$ & Minimum risk of tenant default & & 0.57 \\
\hline Rent review every 4 or more years, upwards only clause & 0.18 & $-0.05^{*}$ & $D \& B 3 A A$ or $4 A A$ rating for tenant(s) & 0.14 & \\
\hline Rent review every 2 to 3 years, no upwards only clause & -0.25 & $0.06^{*}$ & Lower than average risk of tenant default & & 0.41 \\
\hline Rent review every 4 or more years, no upwards only clause & -0.33 & $-0.04 *$ & $\mathrm{D} \& \mathrm{~B} 1 \mathrm{AA}$ or $2 \mathrm{AA}$ rating for tenant(s) & $0.10^{*}$ & \\
\hline NONE & 0.28 & 1.48 & Higher than average risk of tenant default & & -0.21 \\
\hline Consistent Akaike Info Criterion & 1832.01 & 1482.89 & $\mathrm{D} \& \mathrm{~B} A \mathrm{~A}$ or $\mathrm{BB}$ or $\mathrm{CC}$ rating for tenant(s) & $0.00 *$ & \\
\hline Chi Square & 380.6 & 519.27 & D\&B DD or lower rating for tenant(s) & -0.57 & \\
\hline Relative Chi Square & 15.22 & 19.97 & High risk of tenant default & & -0.77 \\
\hline
\end{tabular}




\section{Attribute Preferences (based on HB Estimations)}

\begin{tabular}{|c|c|c|c|c|c|c|c|c|}
\hline & 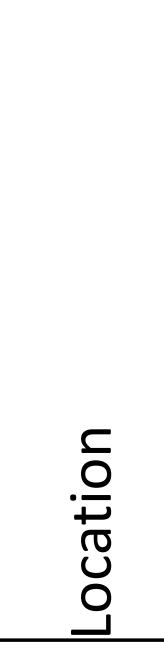 & 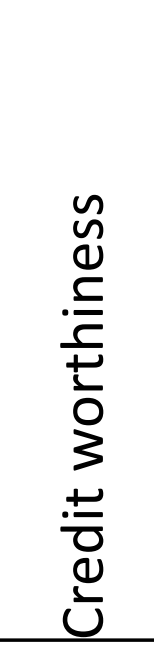 & 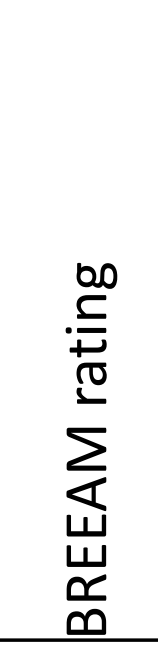 & 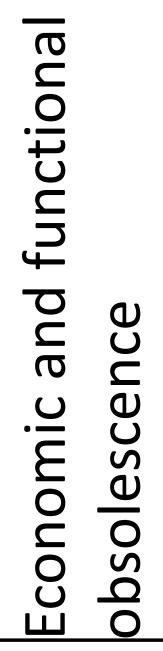 & 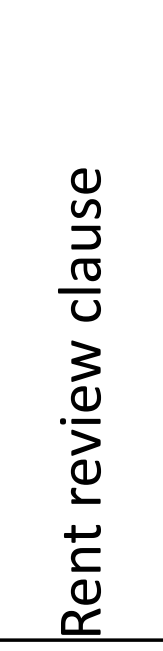 & 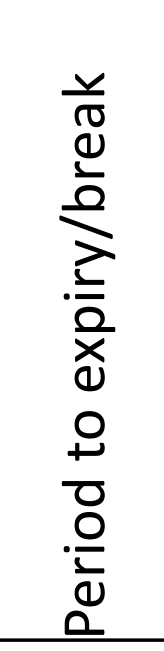 & 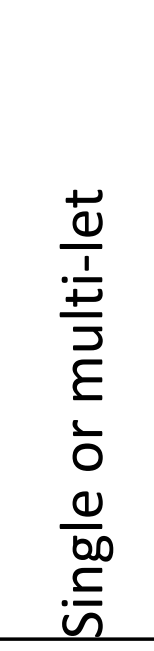 & 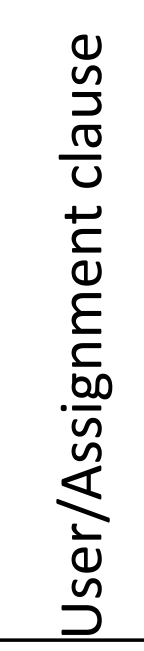 \\
\hline All & 26.33 & 18.48 & 16.56 & 11.18 & 7.91 & 7.55 & 6.55 & 5.44 \\
\hline Core/Core+ & 27.50 & 17.76 & 15.63 & 13.00 & 7.38 & 8.50 & 4.73 & 5.49 \\
\hline Value Added & 20.35 & 23.15 & 16.93 & 8.36 & 7.51 & 16.50 & 4.08 & 3.12 \\
\hline Opportunisic & 11.79 & 16.65 & 14.96 & 12.18 & 15.30 & 11.12 & 11.43 & 6.58 \\
\hline Other & 16.56 & 24.52 & 15.68 & 3.83 & 14.94 & 4.58 & 11.07 & 8.83 \\
\hline Retail & 22.51 & 13.63 & 22.35 & 11.15 & 9.01 & 11.21 & 4.63 & 5.49 \\
\hline Office & 27.15 & 18.15 & 13.44 & 8.97 & 12.53 & 7.24 & 5.72 & 6.79 \\
\hline Industrial & 19.11 & 13.53 & 13.79 & 20.33 & 10.59 & 11.86 & 7.61 & 3.17 \\
\hline Residential & 19.22 & 20.76 & 13.89 & 14.68 & 14.92 & 4.25 & 4.36 & 7.92 \\
\hline Mixed & 19.95 & 26.38 & 11.85 & 11.71 & 11.27 & 3.20 & 3.87 & 11.77 \\
\hline
\end{tabular}




\section{Findings}

$\square$ Ranking of key parameters

- Location still top priority

- BREEAM rating rising to third place

- Credit worthiness

- Economic and functional obsolescence dropped (level of spec and flexibility of internal configuration)

- Period to expiry/number of users/user clauses \& rent review clauses are all insignificant

$\square$ Differs depending on fund style and property type

$\square$ Most preferred BREEAM rating: 'Very Good' 


\section{Conclusions ... so far}

$\square$ Results seem to conform to our a priori expectations

But need to disaggregate further: e.g. impact of economic outlook 


\section{Conclusions ... so far}

$\square$ Results seem to conform to our a priori expectations

$\square$ But need to disaggregate further: e.g. impact of economic outlook

In terms of sustainability:

$\square$ There seems to be a turn in perceptions/preferences

$\square$ Is this linked to fund strategy?

$\square$ Why is 'Very Good' most preferred? Does this change if we disaggregate the sample?

$\square$ When ranked by importance to achieving target returns

- Sustainability rating is $\mathbf{6 . 7 4}$ (out of $\mathbf{8}$ )

$\square$ When ranked by importance to achieving risk mitigation

- Sustainability rating is 6.43 (out of 8 )

(5) RICS

Research

Trust 